\title{
Functional relationship between woody plants and insect communities in response to Bursaphelenchus xylophilus infestation in the Three Gorges Reservoir region
}

\author{
Zhuang Wang ${ }^{1}$, Lijuan Zhao ${ }^{2}$, Jiaqi Liu², Yajie Yang ${ }^{2}$, Juan Shi ${ }^{1}$, Junbao Wen ${ }^{1}$, and Ruihe \\ $\mathrm{GaO}^{2}$ \\ ${ }^{1}$ Beijing Forestry University College of Forestry \\ ${ }^{2}$ Shanxi Agricultural University
}

January 14, 2021

\begin{abstract}
To study the effect of the invasion of Bursaphelenchus xylophilus on the functional relationship between woody plants and insect communities, the populations of tree species and insect communities were investigative in the Masson pine forests with different infestation durations of B. xylophilus. In this study, the number of Pinus massoniana began to decrease sharply, whereas the total number of other tree species in the arboreal layer increased gradually with the infestation duration of B. xylophilus. The principal component analysis ordination biplot shows that there was a significant change in the spatial distribution of woody plant species in different Masson pine forest stands. Additionally, a total of 7,188 insect specimens was obtained. The insect population showed an upward trend in stand types with the increase of pine wilt disease infection periods, which demonstrated that the insect community had been significantly affected by the invasion of B. xylophilus. The structure of insect functional groups changed from herbivorous $(\mathrm{He})>$ omnivorous $(\mathrm{Om})>$ predatory $(\mathrm{Pr})>$ parasitic $(\mathrm{Pa})>\operatorname{detritivorous~}(\mathrm{De})$ in the control stand to $\mathrm{He}>\mathrm{Pa}>\mathrm{Om}$, De $>\mathrm{Pr}$ after B. xylophilus infestation in the forests. The results showed that the populations of $\mathrm{He}, \mathrm{Pa}$, and De increased after the invasion of B. xylophilus, but the populations of Pr decreased. Moreover, the redundancy analysis ordination bi-plots reflected the complicated functional relationship between woody plant communities and insects after the invasion of B. xylophilus. The present study provides insights into the changes in the community structure of woody plants and insects, as well as the functional relationship between woody plant communities and insect communities after invasion of B. xylophilus.
\end{abstract}

Functional relationship between woody plants and insect communities in response to Bursaphelenchus xylophilus infestation in the Three Gorges Reservoir region

Zhuang Wang ${ }^{1 \&}$, Lijuan Zhao ${ }^{2 \&}$, Jiaqi $\mathrm{Liu}^{3}$, Yajie Yang ${ }^{3}$, Juan $\mathrm{Shi}^{1}$, Junbao Wen ${ }^{1 *}$ and Ruihe Gao ${ }^{34 *}$

${ }^{1}$ Beijing Key Laboratory for Forest Pest Control, College of Forestry, Beijing Forestry University, Beijing 100083, China; ${ }^{2}$ College of Marxism, Shanxi Agricultural University, Taigu, Shanxi, 030801, China; ${ }^{3}$ College of Forestry, Shanxi Agricultural University, Taigu, Shanxi, 030801, China; ${ }^{4}$ Shanxi Dangerous Forest Pest Inspection and Identification Center, Taigu, Shanxi, 030801, China

\& The authors contributed equally to this work.

\section{Correspondence}

*Junbao Wen, Beijing Key Laboratory of Forest Pest Control, College of Forestry, Beijing Forestry University, P.O. Box 113, 35 Qinghua East Road, Haidian District, Beijing 100083, P. R. China. Tel: +86-10-62336842; Fax: +86-10-62336842; E-mail: wenjb@bjfu.edu.cn. 
${ }^{*}$ Ruihe Gao, Department of Forest Conservation, College of Forestry, Shanxi Agricultural University, $1^{\text {st }}$ Mingxian Road, Taigu District, Jinzhong, Shanxi Province, P. R. China. Tel: +86-354-6285109; Fax: +86354-6285109; E-mail: gaoruihe1989@163.com.

\title{
Running Head
}

Functional relationship between plant and insect.

\section{Funding information}

This study was supported by the Scientific and Technological Innovation Programs of Higher Education Institutions in Shanxi (Grant No. 2019L0370) and the Technology Innovation Fund of Shanxi Agricultural University (Grant No. 2017YJ20).

\begin{abstract}
To study the effect of the invasion of Bursaphelenchus xylophilus on the functional relationship between woody plants and insect communities, the populations of tree species and insect communities were investigative in the Masson pine forests with different infestation durations of B. xylophilus . In this study, the number of Pinus massoniana began to decrease sharply, whereas the total number of other tree species in the arboreal layer increased gradually with the infestation duration of $B$. xylophilus. The principal component analysis ordination biplot shows that there was a significant change in the spatial distribution of woody plant species in different Masson pine forest stands. Additionally, a total of 7,188 insect specimens was obtained. The insect population showed an upward trend in stand types with the increase of pine wilt disease infection periods, which demonstrated that the insect community had been significantly affected by the invasion of B. xylophilus. The structure of insect functional groups changed from herbivorous (He) $>$ omnivorous $(\mathrm{Om})>$ predatory $(\mathrm{Pr})>$ parasitic $(\mathrm{Pa})>$ detritivorous $(\mathrm{De})$ in the control stand to $\mathrm{He}>\mathrm{Pa}>$ Om, De $>$ Pr after B. xylophilus infestation in the forests. The results showed that the populations of He, $\mathrm{Pa}$, and De increased after the invasion of B.xylophilus, but the populations of Pr decreased. Moreover, the redundancy analysis ordination bi-plots reflected the complicated functional relationship between woody plant communities and insects after the invasion of B. xylophilus. The present study provides insights into the changes in the community structure of woody plants and insects, as well as the functional relationship between woody plant communities and insect communities after invasion of B. xylophilus .
\end{abstract}

\section{KEYWORDS}

Bursaphelenchus xylophilus, plant community structure, insect functional group, functional relationship

\section{INTRODUCTION}

With increase in the frequency of international trade, some invasive alien species are directly or indirectly introduced into new forest ecosystems. As an external disturbance factor, they inevitably affect the productivity, nutrient cycle, hydrological system, species diversity, and information transmission of the new ecosystem (Lovett et al., 2006; Ding et al., 2008; Westphal et al., 2008). The plant community structure and composition of the forest ecosystem can be significantly changed, and the succession rate of the community can be accelerated after the infestation of forestry invasive alien species (Castello et al., 1995; Spiegel \& Leege, 2013; Gao et al., 2015). Consequently, the species composition and population structure of the specific animals, insects, and microorganisms that depend on the plant community may also undergo considerable changes (Karban, 2011; Li et al., 2016; Visakorpi et al., 2019).

As one of the most dangerous and destructive forest pests,Bursaphelenchus xylophilus (Steiner and Bhrer) Nickle (Nematoda: Aphelenchoididae) causes the occurrence of pine wilt disease (PWD) and has a strong destructive effect on pine forest ecosystems in China.Monochamus alternatus is the most efficient insect vector for spreading B. xylophilus. The average number of B. xylophilus carried by M. alternatus is 18,000 per beetle, and the maximum number is 289, 000 (Zhao, 2008). In China, B. xylophilus was first discovered in 40 black pines in September 1982 at Sun Yat-Sen's Mausoleum in Nanjing (Wan et al., 2005; Zhao, 2008). Since then, the degree of damage inflicted by B. xylophilus is a continuous large-scale occurrence, which 
has caused huge economic and ecological losses around China (Wan et al., 2005; Zhao, 2008; Shi et al., 2013). By August 2020, PWD had spread to 18 provinces and 666 counties in China (The No.4 and No.8 bulletin of National Forestry and Grassland Administration in 2020). In addition, the extent of this disease in China has displayed explosive growth in the past two years, with 283 and 85 new counties affected with PWD epidemic added in 2018 and 2019, respectively. Moreover, the outbreak area of PWD has crossed the threshold of annual average temperature above $10^{\circ} \mathrm{C}$, as proposed by the traditional theory (Roques et al., 2015; Firmino et al., 2017; Calvão et al., 2019). The western infection area of B. xylophilushas reached Xichang City, Sichuan Province, and the northern part has colonized Kaiyuan City, Liaoning Province, directly threatening the safety of pine forest resources in northern China (Figure 1).

Previously published works have documented that plant succession is a major factor that affects the insect community's composition and structure (Siemann, 1998; June et al., 2006). As an important environmental indicator, insect species are usually linked to plant communities and are widespread in forest ecosystems (Siemann, 1998; Vandewalle et al., 2010; Cédric et al., 2013). Woody plant species provide much of the habitat and resource base for insects (Brown et al., 2001; Trotter et al., 2008). A greater diversity of plants provides a greater diversity of resources for insects; consequently, the increase in plant species richness also leads to an increase in insect species richness (Knops et al., 1999; Haddad et al., 2001). Furthermore, a higher diversity of herbivorous insects can provide more food sources for insect predators and parasitoids (Knops et al., 1999; Gao et al., 2008).

For the dominant tree species with a large biogeographic distribution, changes in the communities and population structure of this species are likely to affect many other tree or animal species at large landscape scales (Trotter et al., 2008). Masson pine is an important pine tree species in the Three Gorges Reservoir area, which is widely distributed from the bank of the Yangtze River to the top of the mountain (Gao et al., 2015). In this area, the main coniferous forest type was the Masson pine pure forest before the invasion of $B$. xylophilus in 2006. In the following years, this epidemic spread rapidly, killing Masson pine trees occupying a large area.

To date, little is known about the functional relationship between woody plants and insect communities in response to B. xylophilusinfestation. To address this issue, the research of woody plant and insect communities was conducted in the eastern part of the Three Gorges Reservoir region of China. The aim of this work was to address the following questions: after the invasion of B. xylophilus (i) How do woody plant species change? (ii) What impact does the change in woody plant structure have on the insect community structure and composition? (iii) How is the functional relationship between the woody plant community and insects impacted?

\section{MATERIALS AND METHODS}

\subsection{Study sites}

This study was carried out in Yiling District (latitude $30^{\circ} 32^{\prime}-31 \mathrm{deg} 28^{\prime} \mathrm{N}$, longitude 110deg51'-111deg39'E), which located in the eastern part of the Three Gorges Reservoir region and contained the demarcation point of the upper and middle reaches of the Yangtze River (Gao et al., 2019). In this area, PWD was first detected on Masson pine in 2006. Local forestry authorities removed all infected Masson pine trees in 2012, from then the coniferous forest regenerate naturally. The research was conducted on five Masson pine stand types, which were classified based on the duration of PWD infection from 2006 to 2012 (Table 1). Additionally, each Masson pine stand type had three repeated stands, and there were three permanent $15 \mathrm{~m} \mathrm{x} 15 \mathrm{~m}$ Masson pine plots in each stand.

\subsection{Field surveys}

From July to August 2012, the woody plants in the arboreal layer were investigated in each of the 45 plots. For each plot, the basic environmental factors, including elevation, slope, and canopy density, were recorded. The woody plants with a diameter at breast height (DBH) [?] $2.5 \mathrm{~cm}$ were investigated using the method of "Tally" which includes species name, height, DBH, and crown. 


\subsection{Insect sampling and specimen identification}

For each plot, insect sampling was conducted once every 7 days from June to August in 2013 and 2014. Insect specimens were collected by means of "sweep net sampling", "Malaise trap sampling", and "window trap sampling". For the "sweep net sampling" method, a muslin insect swept net was used for sweeping in each corner of the 45 plots, for a total of more than 200 times net was swept for each plot. For the "Malaise trap sampling", a Malaise trap was placed in the center of the 45 plots and was mainly used to trap small insects. For this method, the collected insect specimens could be directly stored in $75 \%$ alcohol, which ensured the necessary quality for subsequent molecular identification. Additionally, five window traps $(29.5 \mathrm{~cm} \times 19.0 \mathrm{~cm} \times 0.2 \mathrm{~cm})$ were placed at the center and the four end points according to the "five-point sampling" method. The window traps were suspended at a height of approximately $3 \mathrm{~m}$, and the collection device was filled with $75 \%$ alcohol in order to preserve the collected insect species.

According to relevant professional books and references, insect individuals were identified as exactly as possible to genus and species. The sampled insect specimens were divided into five groups: herbivorous insects (He), predatory insects $(\mathrm{Pr})$, parasitic insects $(\mathrm{Pa})$, omnivorous insects $(\mathrm{Om})$, and detritivorous insects (De). Moreover, the dominant insect group was the one whose individual number accounted for more than $10 \%$ of the total collected insects, common groups accounted for $1.0 \%-10 \%$ of the total number, and the rare groups accounted for less than 1\% of the total number (Wang and Wang, 2010).

\subsection{Data analysis}

One-way ANOVA and Fisher's least significant difference (LSD) test with an alpha value of $P<0.05$ were used to compare the changes in woody plant and insect community structure. Principal component analysis (PCA) was conducted using CANOCO 5.0 (Microcomputer Power, Ithaca, NY, USA) to determine the plant community structure in different Masson pine stands. In CANOCO 5.0, redundancy analysis (RDA) based on a constrained linear model was used to analyze the ordination relation of woody plant species and insect species. All statistical analyses were performed using GraphPad Prism 6.0 (GraphPad Software, La Jolla, CA, USA) and SPSS 22.0 for Windows (SPSS Inc., Chicago, IL, USA).

\section{RESULTS}

3.1 Changes in woody plant community structure

The total number of $P$. massoniana in the healthy ecosystem (ST1) was higher than that in the infected stand types. With the increase in the infestation duration of PWD, the number of $P$. massonianabegan to decrease sharply, and the statistical difference between different stand types reached a significant level $(F=6.99, P<0.01)$. The total number of other tree species in the arboreal layer (except $P$. massoniana ) increased slowly with the increase in the degree of PWD infestation in the ecosystem. Additionally, the difference in the total number of the remaining species, ST4 and ST5, reached a significant level when compared with the healthy Masson pine ecosystem $(P<0.05)$.

The PCA ordination biplot of woody plant species with an importance value over 0.5 showed that there was a significant change in the structure of the tree layer in different Masson pine forest stands (Figure 2). $P$. massoniana was the most important tree in the tree layer in ST1, with an importance value of up to $67.23 \%$. With the increase in the duration of B. xylophilus infestation, the importance value of $P$. massoniana in the corresponding stand types decreased sharply to $53.61 \%, 50.24 \%, 42.65 \%, 29.24 \%$ in ST2-5. On the contrary, the importance value of Cinnamomum camphora, Quercus aliena, Quercus variabilis, and Loropetalum chinensis showed a rapid upward trend. After 7 years of continuousB. xylophilus infestation, C. camphora had surpassed $P$. massoniana to become the most important tree species in the tree layer in ST5.

\subsection{Insect community structure}

In this study, we collected and identified 7,188 individual insects, representing 510 species from 15 orders and 152 families (Table 3). There were significant differences in the levels of order, family, and individual among the collected insects. Overall, the dominant insect communities were Hemiptera, Coleoptera, Hymenoptera, 
Diptera, and Lepidoptera, which made up more than $10 \%$ of the total at the levels of family, species, and individuals. Other insect communities accounted for a relatively small proportion of the total insect population.

There was an upward trend in the insect population at the levels of order, family, species, and individual among different stand types (Table 4). However, there was little variation in insect community composition at the order, family, and species levels among different stand types, and the difference between them were not significant. Conversely, the individuals were significantly different among different stand types $(F=204.20$, $P$ ¡0.01), and the difference between ST1-2 and ST3-5 was significant.

Overall, the orders of Hemiptera, Coleoptera, Hymenoptera, and Diptera were the dominant insect groups in different Masson pine forest stand types, and each of their relative abundance was all greater than $10 \%$ (Table 5). The dominant insect groups in Hemiptera were Cicadellidae (17.42\%), Aphidoidea (17.07\%), Pentatomidae (15.36\%). The dominant insect groups in Coleoptera were Scarabaeidae (19.08\%). The dominant insect groups in Hymenoptera were Formicidae (37.29\%), Ichneumonidae (14.62\%), and Vespidae (11.26\%). The dominant insect groups in Diptera were Tipulidae (33.91\%) and Cecidomyiidae (14.92\%).

\subsection{Insect functional groups}

The structure of insect functional groups had changed over the course of $B$. xylophilus infestation (Table 6). The magnitude of relative abundance for insect functional groups in the control stand type (ST1) was He $>\mathrm{Om}>\mathrm{Pr}>\mathrm{Pa}>\mathrm{De}$, and after infection, the magnitude of the relative abundance for insect functional groups had changed (ST2-ST5: He $>\mathrm{Pa}>\mathrm{Om}$, De $>\mathrm{Pr}$ ). After the invasion of B. xylophilus, each of the relative abundance of $\mathrm{He}, \mathrm{Pa}$, and $\mathrm{De}$ was increased by varying degrees, with the values of $\mathrm{Pa}$ and $\mathrm{De}$ increasing from $7.86 \%$ to $16.69 \%$ and from $2.10 \%$ to $11.16 \%$, respectively. Meanwhile, the values of the relative abundance of Pr and Om showed a downward trend.

Figure 3 shows the difference in insect functional groups at the levels of species and individuals in different Masson pine forest stands. In terms of He, the number of insect species and individuals increased with the increase in invasion duration of B. xylophilus, and the difference in individuals among different stand types reached a significant level (Figure 3a). For $\mathrm{Pa}$ and De, the number of individuals increased significantly, but the variation range of species was not large (Figure 3b and Figure 3e). There was little variation in the species and individuals of Pr among different stand types (Figure 3c). Both the number of species and individuals of Om decreased when the Masson pine forest was first infested by B. xylophilus, but the number of insect species and individuals demonstrated an increasing trend, and the difference in individual numbers among different stand types reached a significant level (Figure 3d).

The PCA ordination biplot of insect functional groups in different Masson pine forest stands is shown in Figure 4, which intuitively reflects the distribution of the insect community in each stand type. Pr-S was positively associated with ST1 and ST2 but were negatively associated with ST3-ST5. The most important insect functional groups in ST3 and ST5 were Pa-S, Pa-I, Om-S, Om-I, and He-I. However, the He-S, De-S, De-I, and Pr-I groups played essential roles in ST4.

\subsection{Functional relationship between woody plants and insects}

The RDA ordination biplot with woody plant species and insect functional groups along the first two axes is presented in Figure 5. As revealed by the ordination biplot, Pr-S was positively associated with the distribution of P. massoniana and Ilex cornuta in ST1 and ST2. De-S and Pr-I were positively associated with the growth of some broad-leaved tree species, including Q. aliena, Q. variabilis, Loropetalum chinensis , Celtis bungeana ,Rhus typhina, and Symplocos caudatahe. The distribution of Trachycarpus fortune, Cotinus coggygria, andDalbergia hupeana may promote an increase in the numbers of He-S, He-I, Om-S, and De-I. Moreover, there was a significant negative correlation between parasitic insects (Pa-S and $\mathrm{Pa}-\mathrm{I})$ and the distribution of $P$. massoniana, but the parasitic insects were positively associated with some broadleaved tree species, including C. camphora, T. fortune, C. coggygria, D. hupeana, Rhus chinensis, Litsea cubeba, Symplocos paniculate, and Albizia kalkora. 


\section{DISCUSSION}

The present study provides new insights into the functional relationship between woody plants and insect communities after the invasion of $B$. xylophilus. This study mainly examined the community structure and abundance of woody plants and insects in response toB.xylophilus infestation in the eastern part of the Three Gorges Reservoir region of China. The research was conducted on five Masson pine stand types, which were classified based on the duration of PWD infection from 2006 to 2012. Local forestry authorities removed all infected Masson pine trees in 2012, after which the coniferous forest regenerated naturally.

Under natural conditions, B. xylophilus infestation will cause serious damage and loss to a healthy pine forest ecosystem, but with the continuous succession and natural recovery of the ecosystem, the species diversity of the plant community will increase significantly, and the pine forest will evolve and develop in a more advanced direction (Zhao, 2008; Spiegel \& Leege, 2013; Shi et al., 2013). The results of this study show that the invasion of $B$. xylophilus can affect the structure composition of woody plants and significantly affect the spatial and structural distribution of plant species in the arboreal layer. Specifically, the number of stems for all woody tree species measured in five Masson pine forest plots were infected for different periods by B. xylophilus. With the increase in the infestation duration of B. xylophilus, the number of $P$. massonianabegan to decrease sharply. Meanwhile, the total number of other tree species in the arboreal layer (except $P$. massoniana) increased gradually with an increase in the degree of B. xylophilusinfestation in the ecosystem. Similar results were found in many previous studies, which showed that the invasion of B. xylophiluscan lead to the succession of the pure P. massoniana forest ecosystem into a mixed coniferous and broadleaf forest ecosystem or even a broadleaf forest ecosystem (Shi et al., 2007; Wang et al., 2014; Gao et al., 2015). By comparing and analyzing the niche indices of the main woody plant species, the vegetation communities in the woody plant layer did not degenerate in the direction of the shrub layer after the invasion of the Masson pine ecosystem by B. xylophilus . On the contrary, plant species and diversity were greatly increased in the new ecosystem (Wu et al., 2005; Spiegel \& Leege, 2013). In general, the structure of new forest ecosystems formed by succession tends to be more stable and has a strong ability to resist forest pests and diseases (Humphrey et al., 1999; Hamback et al., 2000; Jobidon et al., 2004; Li et al., 2012).

To study the effect of the invasion of B. xylophilus on insects, insect communities were investigated and sampled from five Masson pine stand types with different durations of B. xylophilus infection. In this study, a total of 7,188 individual insects was collected and identified, representing 510 species from 15 orders and 152 families. Overall, the orders of Hemiptera, Coleoptera, Hymenoptera, and Diptera were the dominant insect groups in different Masson pine forest stand types, each with a relative abundance greater than $10 \%$. This finding is consistent with that of a previous study in which the insects of the dominant orders belonged to Coleoptera, Hymenoptera, and Diptera in a coniferous forest ecosystem affected by B. xylophilus in Zhejiang province (Gao et al., 2013). Additionally, the insect population showed an upward trend in Masson pine forest stand types with increasing PWD infection periods, which demonstrated that the insect community was significantly affected by the invasion of B. xylophilus . Previous studies have indicated that a greater diversity of woody plant species provides much more habitats and resources for insects (Root et al., 1973; Siemann, 1998; Brown et al., 2001; Trotter et al., 2008; Vandewalle et al., 2010; Cedric et al., 2013). Therefore, through this study, we found that the invasion of B. xylophilus can affect the community structure and composition, change the spatial and structural distribution of woody plant species, and further affect the distribution of insect community structure and individual populations.

As a crucial part of the biological communities in forest ecosystems, the population dynamics and spatial distribution of insect functional groups are significantly restricted by woody plant species (Root et al., 1973; Vandewalle et al., 2010; Cedric et al., 2013; Bezemer et al., 2014; Tchakonte et al., 2015). The structure of insect functional groups can reflect the development and health status of a specific forest ecosystem (Haddad et al., 2001; June et al., 2006; Karban, 2011; Visakorpi et al., 2019). In this study, the magnitude of relative abundance for insect functional groups in the control stand type (ST1) was He $>\mathrm{Om}>\mathrm{Pr}>\mathrm{Pa}>\mathrm{De}$, whereas after infection, the magnitude of relative abundance for insect functional groups was altered (ST2ST5: $\mathrm{He}>\mathrm{Pa}>\mathrm{Om}, \mathrm{De}>\mathrm{Pr}$ ). This result indicated that the structure of insect functional groups changed 
over the course of B. xylophilus infestation in the Masson pine forests. Moreover, the number of insect species and individuals of herbivorous insects increased with an increase in the invasion duration of B. xylophilus, and the difference of individuals reached a significant level among stand types. This result is consistent with the "Resource Concentration Hypothesis" which states that plant diversity affects the structure of insect functional groups and can significantly increase the species and individual numbers of herbivorous insect populations (Root, 1973). In order to control the population of herbivorous insects and enhance degradation of plant and insect residues (Haddad et al., 2001; June et al., 2006; Trotter et al., 2008; Visakorpi et al., 2019), the relative abundance of parasitic insects and detritivorous insects in this study also increased by varying degrees after the invasion of B. xylophilus, with values increasing from $7.86 \%$ to $16.69 \%$ and from $2.10 \%$ to $11.16 \%$, respectively.

For a more intuitive understanding of the complicated functional relationship between woody plant community and insect functional groups, a redundancy analysis was conducted using 16 woody plant species with an importance value of over 1 , and five insect functional groups with the numbers of species and individuals. As revealed by the RDA ordination graph, the population spatial distribution of insect functional groups was significantly restricted by woody plant species. The numbers of individuals and species of parasitic insects were significantly associated with the distribution of $P$. massoniana, but were positively associated with the distribution of some broad-leaved tree species, which indicated that broad-leaved tree species may provide more resources and ecological niches for parasitic insects. This result is consistent with a previous study showing that the number and diversity index of parasitic insects increased with an increase in the proportion of broad-leaved tree species in a coniferous forest ecosystem in Zhejiang province [36]. Plant diversity restricts the population of parasitic insects and the increase in plant species diversity can provide more habitats and food resources, which can significantly increase the species and individuals of parasitic insects (Knops et al., 1999; Taki et al., 2010; Gao et al., 2013; Gao et al., 2018). As the population of parasitic and predatory insects continues to increase, the number of herbivores, including M. alternatus , which is the vector of B. xylophilus, may be limited.

\section{CONCLUSIONS}

The invasion of B. xylophilus can affect the composition of woody plant communities and change the spatial and structural distribution of woody plant species. A total of 7,188 insect specimens (representing 510 species from 15 orders and 152 families) was obtained and the insect community was significantly affected by the invasion of B. xylophilus. The structure of insect functional groups changed from $\mathrm{He}>\mathrm{Om}>\mathrm{Pr}$ $>\mathrm{Pa}>\mathrm{De}$ in the control stand to $\mathrm{He}>\mathrm{Pa}>\mathrm{Om}$, De $>\mathrm{Pr}$ over the course of B. xylophilus infestation in the Masson pine forests. Moreover, the redundancy analysis ordination bi-plots reflected the complicated functional relationship between the woody plant community and insects after the invasion of B. xylophilus, and the invasion of B. xylophilus can promote the population growth of herbivorous insects, parasitic insects, and detritivorous insects.

\section{ACKNOWLEDFMENTS}

Special thanks go to the anonymous reviewers for their valuable comments and suggestions. We also thank Dewen Song and Kezhou He for their assistance with the collection of data. We used Editage (www.editage.com) for English language editing.

\section{CONFLICT OF INTEREST}

The authors have no conflicts of interest to declared.

\section{AUTHOR CONTRIBUTIONS}

Zhuang Wang : Conceptualization (equal); Data curation (equal); Writing-original draft (equal); Writingreview and editing (equal).Lijuan Zhao : Conceptualization (equal); Data curation (equal); Writing-original draft (equal); Writing-review and editing (equal).Jiaqi Liu : Formal analysis (equal); Software (equal).Yajie Yang : Formal analysis (equal); Software (equal).Juan Shi : Writing-review and editing (equal). Junbao 
Wen : Funding acquisition (equal); Writing-review and editing (equal).Ruihe Gao : Conceptualization (equal); Funding acquisition (equal); Writing-original draft (equal); Writing-review and editing (equal).

\section{DATA AVAILABILITY STATEMENT}

The data are available in the Dryad database under the following link: https://doi.org/10.5061/dryad.c59zw3r6d

\section{ORCID}

Ruihe Gao https://orcid.org/0000-0001-7447-5648

\section{REFERENCES}

Bezemer, T.M., Harvey, J.A., \& Cronin, J.T. (2014). Response of native insect communities to invasive plants. Annual Review of Entomology , 59 , 119-141.

Brown, J.H., Whitham, T.G., Ernest, S.K.M., \& Gehring, C.A. (2001). Complex species interactions and the dynamics of ecological systems: long-term experiments. Science , 293 , 643-650.

Calvao, T., Duarte, C.M., \& Pimentel, C.S. (2019). Climate and landscape patterns of pine forest decline after invasion by the pinewood nematode. Forest Ecology and Management , 433 , 43-51.

Castello, J.D., Leopold, D.J., \& Smallidge, P.J. (1995). Pathogens, patterns, and processes in forest ecosystems.Bioscience ,45 , 16-24.

Cedric, F.D., Bill, S., \& Yves, H. (2013). Linking plant and insect traits to understand multitrophic community structure in arid steppes.Functional Ecology , $27,3$.

Ding, J., Mack, R.N., Lu P., Ren, M.X., \& Huang, H.W. (2008). China's booming economy is sparking and accelerating biological invasions.Bioscience, 58 , 317-324.

Firmino, P.N., Calvao, T., Ayres, M.P., \& Pimentel, C.S. (2017).Monochamus galloprovincialis and Bursaphelenchus xylophilus life history in an area severely affected by pine wilt disease: implications for forest management. Forest Ecology and Management, 389 , 105-115.

Gao R.H., Wang, Z., Wang, H.X., Hao, Y.P., \& Shi, J. (2019). Relationship between pine wilt disease outbreaks and climatic variables in the Three Gorges Reservoir Region. Forests , 10 , 9.

Gao, R.H., Luo, Y.Q., Wang, Z., Yu, H.J., \& Shi, J. (2018). Patterns of biomass, carbon, and nitrogen storage distribution dynamics after the invasion of pine forests by Bursaphelenchus xylophilus (Nematoda: Aphelenchoididae) in the three Gorges Reservoir Region. Journal of Forestry Research , 29 , 459-470.

Gao, R.H., Shi, J., Huang, R.F., Wang, Z., \& Luo, Y.Q. (2015). Effects of pine wilt disease invasion on soil properties and Masson pine forest communities in the Three Gorges reservoir region, China. Ecology and Evolution , 5 , 1702-1716.

Gao, R.H., Wang, Z., Shi, J., \& Luo, Y.Q. (2013). Characteristics of insect community and the spread of parasitic insects under environmental gradients in cut slash affected by pine wilt disease. Journal of Beijing Forestry University, 35 , 84-90.

Haddad, N.M., Tilman, D., Haarstad, J., Ritchie, M., \& Knops, J.M.H. (2001). Contrasting effects of plant richness and composition on insect communities: a field experiment. American Naturalist , 158 , 17-35.

Hamback P.A., Agren, J., \& Ericson, L. (2000). Associational resistance: insect damage to purple loosestrife reduced in thickets of sweet gale. Ecology , 81 , 1784-1794.

Humphrey, J., Hawes, C., Peace, A., Ferris-Kaan, R., \& Jukes, M. (1999). Relationships between insect diversity and habitat characteristics in plantation forests. Forest Ecology and Management, 113 , 11-21. 
Jobidon, R., Cyr, G., \& Thiffault, N. (2004). Plant species diversity and composition along an experimental gradient of northern hardwood abundance in Picea mariana plantations. Forest Ecology and Management, $198,209-221$.

June, M.J., Robert, J.M., \& Rebecca, E.F. (2006). Forest age influences oak insect herbivore community structure, richness, and density.Ecological Applications , 16 , 901-912.

Karban, R. (2011). The ecology and evolution of induced resistance against herbivores: induced resistance against herbivores. Functional Ecology ,25, 339-347.

Knops, J.M.H., Tilman, D., Haddad, N.M., Naeem, S., Mitchell, C.E., Haarstad, J., Ritchie, M.E., Howe, K.M., Reich, P.B., Siemann, E., \& Groth, J. (1999). Effects of plant species richness on invasion dynamics, disease outbreaks, and insect abundances and diversity.Ecology Letters , 2 , 286-293.

Li, J., Shi, J., Luo, Y.Q., \& Heliovaara, K. (2012). Plant and insect diversity along an experimental gradient of larch-birch mixtures in Chinese boreal forest.Turkish Journal of Agriculture and Forestry , 36 , 247-255.

Li, Y., Stam, J.M., Poelman, E.H., Dicke, M., \& Gols, R. (2016). Community structure and abundance of insects in response to early-season aphid infestation in wild cabbage populations: community responses to aphid infestation. Ecological Entomology , 41 , 378-388.

Lovett, G.M., Canham, C.D., Arthur, M.A., Weathers, K.C., \& Fitzhugh, R.D. (2006). Forest ecosystem responses to exotic pests and pathogens in eastern North America. BioScience, 56 , 395-405.

Root, R.B. (1973). Organization of a plant-arthropod association in simple and diverse habitats: the fauna of collards (Brassica oleraceae).Ecological Monographs , 43 , 95-124.

Roques, A., Zhao, L.L., Sun, J.H., \& Robinet, C. (2015). Pine wood nematode, pine wilt disease, vector beetle and pine tree: how a multiplayer system could reply to climate change. In Climate Change and Insect Pests ; Bjorkman, C., Niemela, P., Eds.; CABI publishing: Oxfordshire, UK, pp. 220-234. ISBN 9781780643786 .

Shi, J., Chen, F., Luo, Y.Q., Wang, Z., \& Xie, B.Y. (2013). First isolation of pine wood nematode from Pinus tabuliformis forests in China. Forest Pathology ,43 , 59-66.

Shi, J., Luo, Y.Q., Song, J.Y., Wu, H.W., Wang, L., \& Wang, G.Z. (2007). Traits of Masson pine affecting attack of pine wood nematode.Journal of Integrative Plant Biology , 49 , 1763-1771.

Siemann, E. (1998). Experimental tests of effects of plant productivity and diversity on grassland arthropod diversity. Ecology ,79 , 2057-2070.

Spiegel, K.S., \& Leege, L.M. (2013). Impacts of laurel wilt disease on redbay (Persea borbonia (L.) Spreng.) population structure and forest communities in the coastal plain of Georgia, USA.Biological Invasions , 15 , 2467-2487.

Taki, H., Inoue, T., Tanka, H., Makihara, H., Sueyoshi, M., Isono, M., \& Okabe, K. (2010). Responses of community structure, diversity, and abundance of understory plants and insect assemblages to thinning in plantations. Forest Ecology and Management, 259, 607-613.

Tchakonte, S., Ajeagah, G.A., Camara, A.I., Diomande, D., Tchatcho, N.L.N., \& Ngassam, P. (2015). Impact of urbanization on aquatic insect assemblages in the coastal zone of Cameroon: the use of bio-traits and indicator taxa to assess environmental pollution. Hydrobiologia ,755 , 123-144.

The 2020 No.4 bulletin of National Forestry and Grassland Administration-The epidemic area of Pine Wilt Disease. (2020). Available online: http://www.gov.cn/zhengce/zhengceku/2020-03/16/content

-5491788.

The 2020 No.18 bulletin of National Forestry and Grassland Administration-The epidemic area of Pine Wilt Disease. (2020). Available online: http://www.forestry.gov.cn/main/586/20201014/14424644 4501073. 
Trotter, R., Talbot, C., Neil, S., \& Whitham, T.G. (2008). Arthropod community diversity and trophic structure: a comparison between extremes of plant stress. Ecological Entomology , 33 , 1-11.

Vandewalle, M., Bello, F., Berg, M.P., Bolger, T., Doledec, S., Dubs, F., Feld, C.K., Harrington, R., Harrison, S.A., Lavorel, S., Martins, P., Moretti, M., Niemela, J., Santos, P., Sattler, T., Sykes, M.T., Vanbergen, A.J., \& Woodcock, B.A. (2010). Functional traits as indicators of biodiversity response to land use changes across ecosystems and organisms. Biodiversity and Conservation ,19, 2921-2947.

Visakorpi, K., Riutta, T., Martinez, A.E., Salminen, J.P., \& Gripenberg, S. (2019). Insect community structure covaries with host plant chemistry but is not affected by prior herbivory. Ecology ,100 , 8, e02739.

Wan, F., Zheng, X., \& Guo, J. (2005). Biology and management of invasive alien species in agriculture and forestry. Science Publication, Beijing, pp14-19.

Wang, J.F., \& Wang, X.P. (2010). Analysis and evaluation of insect community diversity in Shuidonggou wetland of Ningxia. China Bulletin Entomology , 47, 962-967.

Wang, Z., Luo, Y.Q., Shi, J., Gao, R.H., \& Wang, G.M. (2014). Quantitative classification and environmental interpretation of secondary forests 18 years after the invasion of pine forests byBursaphelenchus xylophilus (Nematoda: Aphelenchoididae) in China.Journal of Insect Science , 14 , 10. 1093.

Westphal, M.I., Browne, M., MacKinnon, K., \& Noble, I. (2008). The link between international trade and the global distribution of invasive alien species. Biological Invasions , 10 , 391-398.

Wu, R., Chen, Y.W., Chen, Z.M., Lin, X.J., \& Liang, D.D. (2005). Effects of pine wood nematode invasion on succession of different pine forest communities. Journal of Southwest Forestry College ,2 , 39-43.

Zhao, B.G. (2008). Pine wilt disease in China. In pine wilt disease; Zhao, B.G., Futai, K., Sutherland, J.R., Takeuchi, Y., Eds.; Springer: Tokyo, Japan, pp. 18-25. ISBN 978-4-431-75655-2.

Figure legends :

Figure 1. The actual epidemic distribution areas of pine wilt disease in China in August 2020. (Data obtained from the No.4 and No.18 bulletin of National Forestry and Grassland Administration in 2020).

Figure 2. Principal component analysis ordination diagram of plant community structure in different Masson pine forest stands.1. Pinus massoniana ; 2. Cinnamomum camphora ; 3 . Quercus aliena ; 4. Quercus variabilis ; 5. Loropetalum chinensis ; 6. Rhus chinensis ; 7. Celtis bungeana ; 8. Trachycarpus fortunei ; 9. Cotinus coggygria ;10. Litsea cubeba ; 11. Symplocos paniculata ; 12. Rhus typhina ; 13.Dalbergia hupeana ; 14. Ilex cornuta ; 15. Albizia kalkora ; 16. Symplocos caudata ;17. Aralia chinensis ; 18. Rhamnus parvifolius ; 19. Castanea mollissima ; 20. Pistacia chinensis ; 21. Deutzia grandiflora ;22. Camellia oleifera ; 23. Melia azedarach ; 24. Sapium sebiferum ; 25.Sabina chinensis ; 26. Remaining species.

Figure 3. The difference of insect functional groups at the level of species and individuals in different Masson pine forest stands. (a ) the difference of insect functional groups for herbivorous insects; (b ) the difference of insect functional groups for parasitic insects; (c ) the difference of insect functional groups for predatory insects; (d ) the difference of insect functional groups for omnivorous insects; (e ) the difference of insect functional groups for detritivorous insects. Mean values of the number of species among different stand types followed by different uppercase letters are significantly different at $P=0.05$ level, mean values of the number of individuals among different stand types followed by different lowercase letters are significantly different at $P=0.05$ level.

Figure 4. Principal component analysis ordination diagram of insect functional groups in different Masson pine forest stands.He-S : Number of species of herbivorous insects; He-I : Number of individuals of herbivorous insects; Pa-S : Number of species of parasitic insects; Pa-I : Number of individuals of parasitic insects; Pr-S : Number of species of predatory insects; Pr-I : Number of individuals of predatory insects; Om-S 
: Number of species of omnivorous insects; Om-I: Number of individuals of omnivorous insects; De-S : Number of species of detritivorous insects; De-I : Number of individuals of detritivorous insects.

Figure 5. Results of the RDA ordination biplot presenting woody plant species and insect functional groups in different Masson pine forest stands. For woody plant species variables: 1.Pinus massoniana $; 2$. Cinnamomum camphora ;3. Quercus aliena; 4. Quercus variabilis; 5. Loropetalum chinensis; 6. Rhus chinensis ; 7. Celtis bungeana ; 8. Trachycarpus fortunei ; 9. Cotinus coggygria ;10. Litsea cubeba ; 11. Symplocos paniculata ; 12. Rhus typhina ; 13.Dalbergia hupeana; 14. Ilex cornuta; 15. Albizia kalkora; 16. Symplocos caudata. For insect functional group variables: He-S : Species of herbivorous insects; He-I : Individuals of herbivorous insects;Pa-S : Species of parasitic insects; Pa-I : Individuals of parasitic insects; Pr-S: Species of predatory insects; Pr-I : Individuals of predatory insects; Om-S : Species of omnivorous insects; Om-I : Individuals of omnivorous insects;De-S : Species of detritivorous insects; De-I : Individuals of detritivorous insects. 


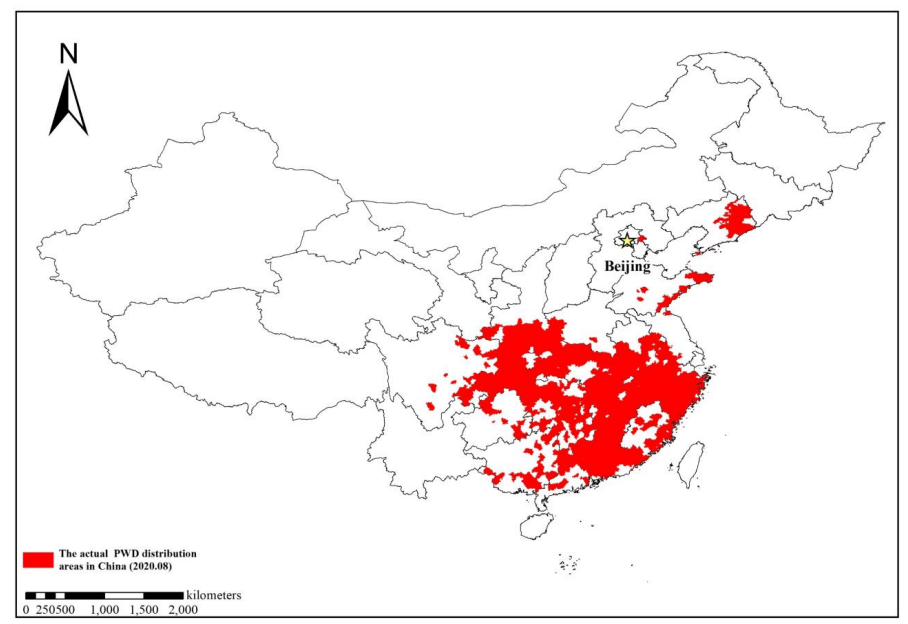




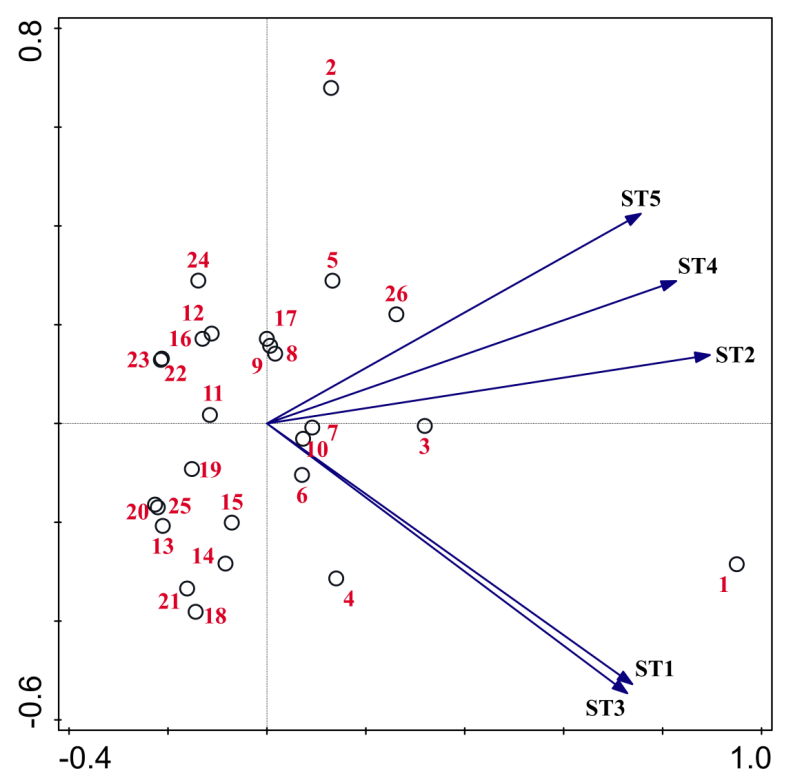




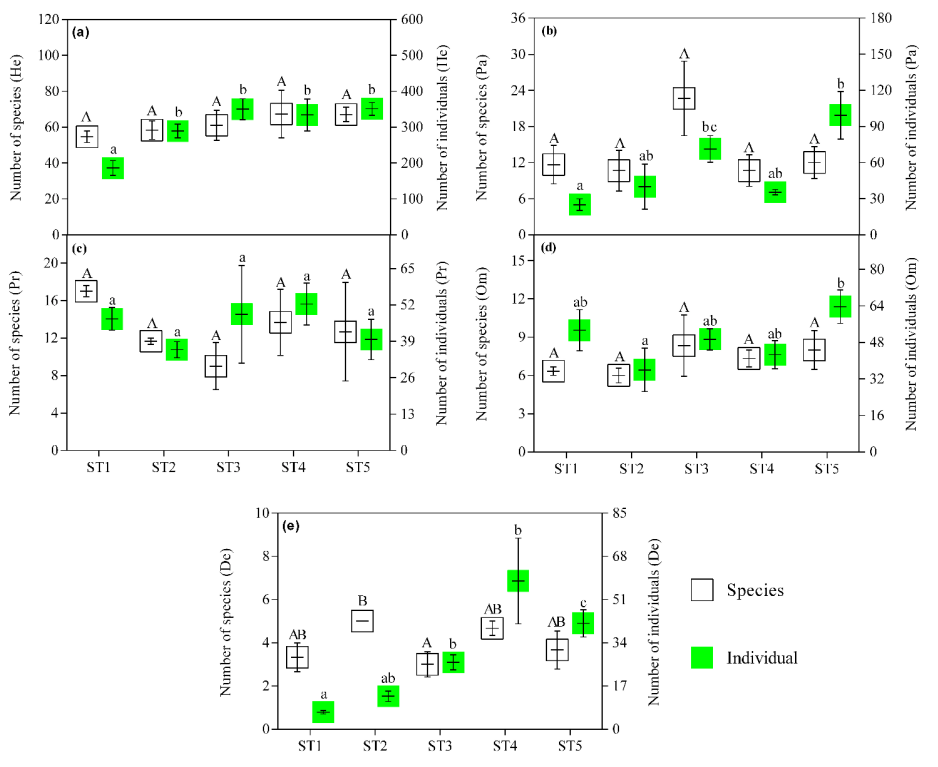




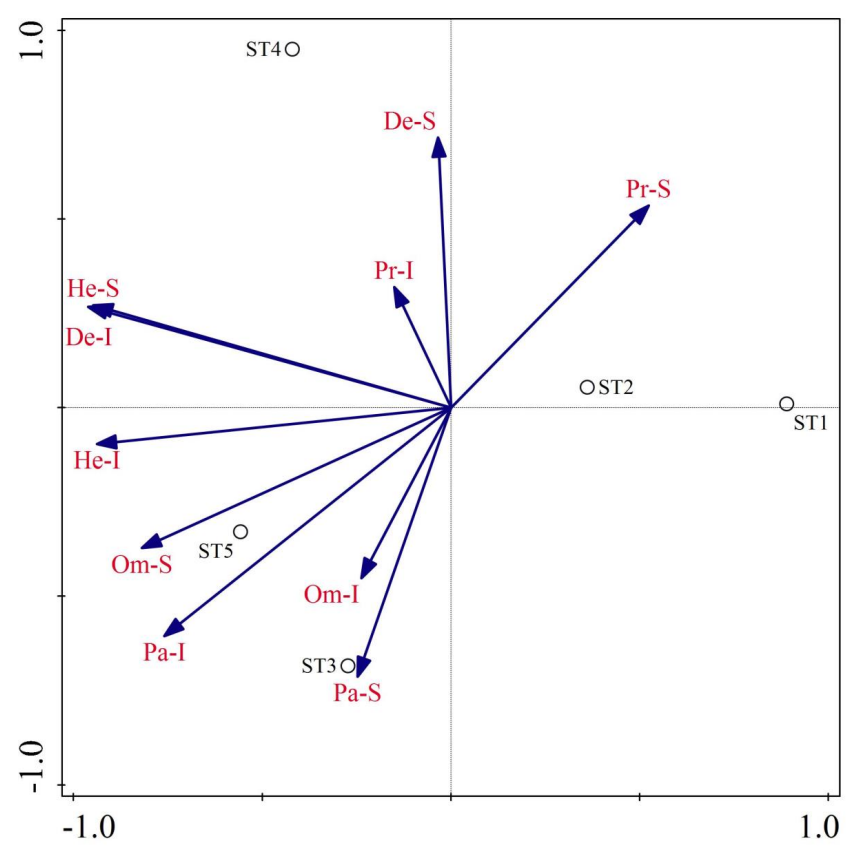




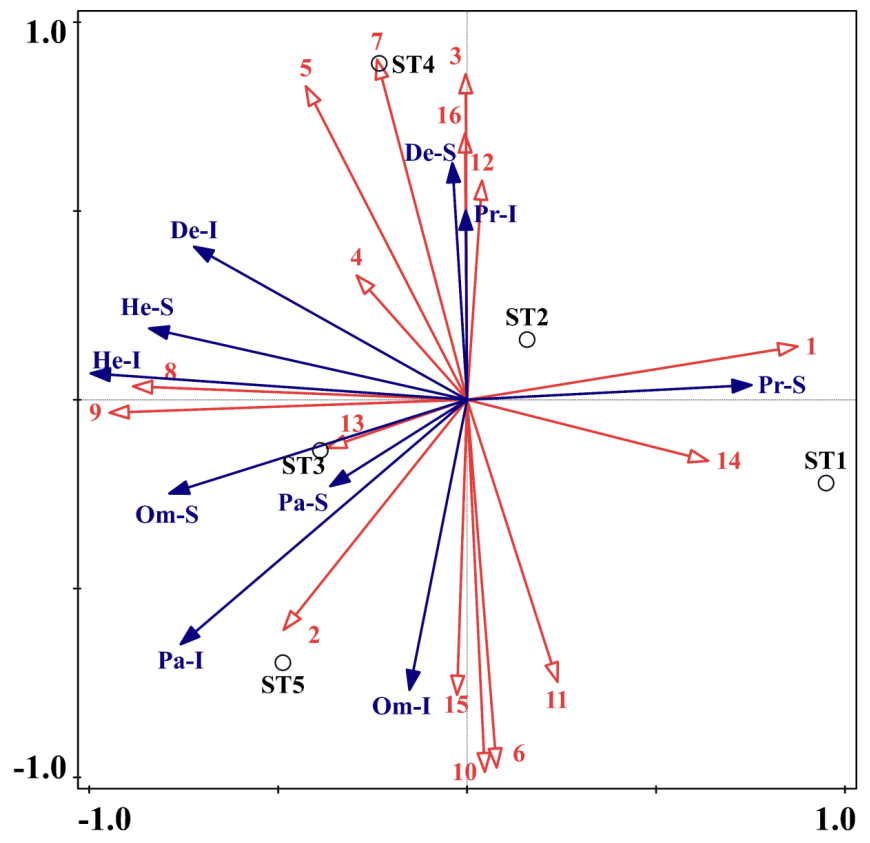

